\title{
Anabases
}

ANABASES Traditions et réceptions de l'Antiquité

$9 \mid 2009$

Varia

\section{Mythologie antique et amours incestueuses : le regard d'un clerc du Moyen Âge}

\section{Marylène Possamaï-Perez}

\section{(2) OpenEdition}

1 Journals

Édition électronique

URL : http://journals.openedition.org/anabases/406

DOI : 10.4000/anabases.406

ISSN : 2256-9421

\section{Éditeur}

E.R.A.S.M.E.

\section{Édition imprimée}

Date de publication : 1 mars 2009

Pagination : 173-184

ISSN : 1774-4296

\section{Référence électronique}

Marylène Possamaï-Perez, " Mythologie antique et amours incestueuses : le regard d'un clerc du Moyen Âge », Anabases [En ligne], 9 | 2009, mis en ligne le 01 mars 2012, consulté le 10 décembre 2020. URL : http://journals.openedition.org/anabases/406 ; DOI : https://doi.org/10.4000/anabases. 406

(c) Anabases 
Anabases 9 (2009), p. 173-184.

\section{Mythologie antique et amours incestueuses: le regard d'un clerc du Moyen Âge}

Marylène Possamai-Pérez

L'AUTEUR DE L' OVIDE MORALISÉ, qui, au début du XIV siècle, « traduit » en langue romane les Métamorphoses d'Ovide, ne craint pas de reprendre les histoires d'amours incestueuses. Comment un penseur chrétien du Moyen Âge peut-il justifier ces récits scandaleux, quelles stratégies peut-il adopter pour les rendre acceptables? Nul doute qu'elles lui posent problème, comme en témoignent les formules récurrentes par lesquelles il s'excuse de les proposer à ses lecteurs. Mais les mots qui précèdent le récit de la légende de Myrrha ${ }^{1}$ suggèrent peut-être une explication: Myrrha fut cruellement punie, et ce châtiment peut contenir la "vérité " de la légende. Le translateur répond donc à des préoccupations morales, qui rejoignent celles des penseurs de tous les temps.

Mais la fascination-répulsion qu'il éprouve à l'égard de ces légendes, qu'il amplifie, a peut-être des raisons plus profondes. La pensée de notre moraliste du XIV se confronte avec les conceptions ovidiennes, qui transparaissent dans la translation de ses fables, avec celles de "l'inconscient collectif " aussi, celles que contiennent les mythes universels. Il rejoint les penseurs de tous les temps qui ont réfléchi sur les relations entre l'inceste et la société humaine.

Cependant l'idéologie chrétienne renverse les conceptions ancestrales, la morale tribale: l'allégorie du XIV siècle réhabilite les amours honteuses, folies, démesures de la légende, dans une vraie révolution - une conversion au sens propre du terme. Si le

1 Ce samble cruel chose à dire / Mes puis qu'il chiet en ma matire / Dire en vueil. Ensus vous traiez, / Filletes, que vous ne l'oiez, / Mes s'il vous delite à savoir, / Ne crees pas cest conte à voir. / Se le crees, si soies certes / Qu'ele en reçut crueuls desertes (O.m., X, 1096-1103). Toutes les citations de l'Ovide moralisé (abréviation O.m.) sont tirées de l'édition C. DE BoER, Amsterdam, 1915-1938. 
moraliste reprend ces légendes d'inceste, c'est parce qu'elles lui permettent de représenter concrètement le miracle du dogme chrétien.

\section{Les amours incestueuses de la fable}

L'inceste est le thème central des légendes de Myrrha ou de Byblis: la première éprouve un désir coupable pour son propre père, la deuxième est amoureuse de son frère. Or l'inceste est condamné de manière quasiment unanime dans toutes les sociétés antiques, médiévales et même modernes. Le translateur du XIV ${ }^{e}$ siècle va-t-il supprimer, réduire, transformer ces récits scandaleux?

L'histoire de Myrrha est une "affreuse histoire ", dira (X, 300), l'une de celles qui nécessitent des « excuses " au lecteur, même chez le cruel Ovide ${ }^{2}:$ " Retirez-vous, jeunes filles; parents, retirez-vous; ou, si mes chants séduisent vos cœurs, n’ajoutez point foi à ce récit; ne croyez pas au fait; ou, si vous y croyez, croyez aussi au châtiment du fait ${ }^{3}$. » Le translateur roman reprend fidèlement cette excuse ${ }^{4}$. La christianisation est à l'œuvre dès la traduction des légendes, puisque nefas (306) devient pechié (1105). Mais dans les deux textes il s'agit bien d'une faute contre nature (304 et 1104). L'auteur chrétien supprime les mentions du dieu Amour et des trois Furies, mais amplifie très largement les réflexions morales sur la passion de Myrrha: les vers 414-415 (scelus est odisse parentem; / Hic amor est odio maius scelus) deviennent une longue tirade (1126-1165) qui oppose ce pechié (1131), cette rage (1140,1152), à l'amour tel que le définit saint Paul dans la Lettre aux Corinthiens (1143-1149). Le désir de Myrrha pour son père dépasse la mesure $(1126,1128,1137)$, s'oppose au droit $(1135,1136,1138)$, est forsen et folie (1151, 1160), honteuse chose (1165). Dans cette longue addition, le translateur se montre cependant fidèle disciple d'Ovide, puisqu'il signale l'impuissance de la victime à lutter contre sa passion (1153-54), et les cruelles souffrances que provoque cette passion $(1155,1156)$. Cet amour est contraire à toutes les lois divines et humaines: l'héroïne latine demande aux dieux, à la piété filiale et aux droits sacrés des parents, de

2 Cf. F. Frontisi, “Ovide pornographe? Comment lire les récits de viol”, Clio 19 (2004), Femmes et image, p. 21-35: "La cruauté du poète, et sa sublime efficacité, ne sont plus à démontrer" (p. 24-25).

Mét., X, 300-303: procul hinc natae, procul este parentes; / Aut, mea si vestras mulcebunt carmina mentes, / Desit in hac mihi parte fides, nec credite factum; / Vel, si credetis, facti quoque credite poenam: c'est l'un des exemples où le poète latin semble douter de la crédibilité des légendes qu'il raconte, et c'est peut-être l'une des raisons de son exil. Toutes les citations des Métamorphoses (abréviation Mét.) sont tirées de l'édition et de la traduction de G. Lafaye, Paris, 1969.

4 On se souvient que cruel chose traduit dira, et que les filletes sont invitées à s'éloigner, ou à ne pas croire que le conte soit vrai, ou du moins à être sûres Qu'ele en reçut crueulz desertes (1092-1103). On le voit, la cruauté est du côté de la faute, elle est aussi celle du châtiment. 
la préserver de l'inceste (nefas), du crime (scelus) (321-322). Son homologue romane se contente de qualifier son désir de forsenerie (1168), de puterie (1169), de felonie (1174). Dans les deux textes, la folie combat la raison et l'héroïne ne craint pas d'envier le comportement sexuel des animaux ${ }^{5}$, ce qui lui permet de retourner l'argument de la nature (Mét., 330, O. m., 1184) et celui des lois: les lois humaines sont méchantes (malignas, 329, invida iura, 231) ${ }^{6}$.

Byblis est présentée comme un contre-exemple pour les jeunes filles, ut ament concessa, pour qu'elles aient des amours " admises", légitimes ${ }^{7}$. Par lui pueent example prendre / Ces damoiseles et apprendre / Qu'eles n'aiment trop folement (O. m., IX, 2079-81). C'est toujours le thème de la passion contraire à la raison: Byblis est " emportée de désir ", correpta cupidine fratris ${ }^{8}$, elle aime son frère outre mesure pour l'Ovide moralisé, qui met l'accent sur l'excès de cette passion (IX, 2083). Pourtant elle ne pense pas commettre une "mauvaiseté »: nec peccare putat (458), n'i entent à manvestié, / Ne pas ne cuide avoir pechié (2085-86). C'est une autre caractéristique de la passion, elle trompe celui même qui en est la victime: Byblis « est abusée par l'apparence mensongère de l'amour familial ${ }^{9}$ "; elle ne peut apercevoir sa desverie (2096: par ce sentiment elle desvoie, elle sort du sillon, de la droite voie, de la norme), Et si n'ose elle en nulle fin / En veillant penser si grant rage / Qu'el face à son frere putage (21002102): sa pensée consciente lui masque la culpabilité de ses sentiments, que le poète nomme rage, comme l'amour de Narcisse, et putage, débauche, péché de luxure. Mais, comme les noms révèlent l'essence des êtres et des choses, elle rejette le nom de frère pour préférer celui de " maître, seigneur », équivalent de " mari, époux »: Iam dominum appellat... (466-67). C'est un songe qui lui révèle la vraie nature de ses sentiments, et au réveil elle en conçoit de la honte, de la souffrance ${ }^{10}$, l'horreur qu'on éprouve devant la "merveille ", la monstruosité, le phénomène contraire à la raison, à la nature: Avis li est qu'ele l'embrace / Et que tous ses talents en face. / De ce se vergonde forment / [...] quant elle s'esveille, / Si s'esbahit mout et merveille / De ce songe... Freud dirait que ce rêve traduit les pulsions de son inconscient: le moi, guidé par le subconscient, en

Mét., X, 324-330, O. m., X, 1179-82.

Nous verrons l'importance de ces dernières remarques: si l'inceste appartient aux « mauvaisetés " sexuelles, c'est qu'il ravale l'homme au rang de la bête. Cf. F. Héritier, "Réflexions pour nourrir la réflexion", De la violence, Paris, 1996, p. 39-40.

Mét., IX, 454.

"Violemment éprise de son frère ", ibid., 455.

Mendacique diu pietatis fallitur umbra, Mét., IX, 460. Le mot umbra est utilisé pour le reflet qui trompe Narcisse.

À vrai dire sa souffrance devient vite celle du désespoir, de la certitude qu'elle ne pourra pas ressentir ce plaisir dans la réalité: Trop me despere, trop m'esmoi, / Quar je ne puis penser que j'oie / De mon frere delit ne joie, O.m., 2158-60. L'amour selon la conception ovidienne, que transcrit le poète médiéval, est maladie, blessure: cf. O.m., 2428-30. 
repousse la réalisation ${ }^{11} \ldots$ mais ne refuse pas de retrouver la "volupté " procurée par ces visions ${ }^{12}$. Cet amour n'est permis qu'aux dieux, il est surhumain, inhumain donc: Sunt superi jura: quid ad caelestia ritus / Exigere humanos diversaque foedera tempto ${ }^{13}$ Le long monologue de l'héroïne traduit une nouvelle fois le dilemme, la douloureuse prise de conscience de la folie, de la démesure, de l'impossibilité de la passion: Giter m'estuet de mon corage / La fole amour qui me court sore, / Ou male mort soit, qui m'acore $(2188-90)^{14}$. Comme Narcisse, Byblis appelle la mort ${ }^{15}$. Cet amour est crime, scelus (506), "flammes impures ", obscenae flammae (509) pour le poète latin, folie, desmesure (2200), rage (2209), amour honteuse et vilz (2211) pour le moraliste médiéval. Ce n'est pas l'amour permis à une sœur, qua fas est germanae (Mét., 510), mais un péché ( $O$. $m ., 2214)$. C'est une passion si violente qu'elle ne peut rester enfermée dans un cour: elle doit éclater, être révélée ${ }^{16}$. La lettre que Byblis écrit à son frère souligne aussi bien la violence irraisonnée de cet amour ${ }^{17}$ que la façon dont il porte atteinte au droit, aux lois, à l'ordre établi que symbolisent les vieillards ${ }^{18}$. La malheureuse espère pouvoir dissimuler la culpabilité de ses sentiments: Dulcia fraterno sub nomine furta tegemus (Mét., 559); l'Ovide moralisé est ici plus explicite: ja nulz ne me tendra por fole, / Se je t'embrace ou je t'acole. / Ja n'i pensera mauvestié (2327-29). Il s'agit bien de " mauvaiseté ", de comportement sexuel contre nature, dangereux pour la cité ${ }^{19}$. Son aspect transgressif est souligné par le présage qui semble envoyé par les dieux (on pense au mariage de Térée et Procné dans la légende de Philomena) : les tablettes de cire du récit latin, la « lettre " de la version romane, tombent quand Byblis les confie à son messager, mais l'héroïne ne tient pas compte de l'avertissement et transgresse l'interdit (Mét., 571-573; O. m., 2355-60). La réaction de Caunus (Cadmus écrit le translateur - mais son " erreur " s'expliquera) est conforme à la raison, à la morale: elle est marquée par la honte, la colère et le dégoût ${ }^{20}$. En l'apprenant, la douleur de Byblis est telle qu'elle subit comme l'annonce d'une métamorphose: Pale devient et de dolour / Perdi le sans

Mét., 475, O. m., 2116-17.

Mét., 481: nec abest imitata voluptas; O. m., 2135: J'ai grant delit en l'avision.

Mét., 500-501: "Les dieux ont leurs privilèges; comment puis-je mesurer les rites des humains d'après les lois toutes différentes qui lient entre eux les immortels? » Cf. O. m., 2184-87.

Cf. 2232-38.

Cf. Mét., 503-504.

coget amor (Mét., 515), Force d'amours mel fera faire (O. m., 2226). On pense à l'aveu de Phèdre.

Cf. Mét., 540 (grave vulnus), 541 (furor igneus), 543 (violentia Cupidinis arma), 562 (ultimus ardor); O. m., 2278-86: l'angoisseuse amours, mon fol corage, n'i vault force ne savoir, Contre amours ne puis force avoir. I Vaincue m'a; ne puis durer / Ne ses assaulz plus endurer.

Cf. Mét., 552-553; O. m., 2298-2302.

Cf. F. Héritier, De la violence, p. 39-40.

Cf. Mét.: ira (574), pudor (578), ferocia dicta (580-81). O. m., 2367-68: Le cuer a moult triste et dolent. / L'escript gita par maltalent; 2378: le fier respons et la grant honte. 
et la couleur. / Plus froide de marbre devint (2381-83) ${ }^{21}$. Mais, dans un deuxième long monologue ${ }^{22}$, l'héroïne tout à la fois analyse la perversité de ses sentiments ${ }^{23}$ et s'obstine à les avouer à son frère, péchant par pertinacia. L'inceste est appelé nefas par Ovide (633), alors que l'auteur médiéval ne l'évoque que par euphémisme (ceste guerre, 2494). Après la fuite de son frère, la malheureuse perd totalement la raison, et s'épuise en larmes qui deviennent fontaine, Sic lacrimis consumpta suis [...] / Vertitur in fontem ${ }^{24}$; Si plore et de plorer ne fine, I Tant qu'en pures lermes decourt. / Sous ses lermes une dois court / De vive et pardurable vaine: / Biblis fu muee en fontaine (O. m., 2522-26).

Le moraliste chrétien n'efface rien du désir criminel des héroïnes. Au contraire, il développe leurs sentiments honteux dans de longues tirades.

Ainsi donc le translateur roman se complaît à restituer les légendes antiques d'amours incestueuses. Si l'on ne peut nier qu'il y prend plaisir $^{25}$, il est probable aussi que, comme tout écrivain médiéval, les préoccupations morales ne sont pas absentes de son propos ${ }^{26}$, comme elles ne l'étaient pas, sans doute, du dessein de son modèle antique.

\section{La condamnation morale des légendes}

Se l'escripture ne me ment, / Tout est pour nostre enseignement / Quanqu'il a es livre escript, / Soient bon ou mal li escript. / Li maulz y est que l'en s'en gart (I, 1-5). La peinture des fautes sexuelles aura une valeur exemplaire aussi grande que celle des nobles comportements, "les récits contiennent le mal pour que l'on s'en préserve ".

La condamnation des " mauvaisetés sexuelles " n'est pas réservée à la société médiévale: elle est universelle, comme le constate F. Héritier ${ }^{27}$, qui repart des mythes grecs pour son étude de l'inceste. "La mauvaise sexualité humaine entraîne saturation de l'espace, sécheresse et infertilité, comme elle entraînait le loimos chez les Grecs. » Le loimos, le fléau, la peste qui ravage l'armée grecque devant Troie ou Thèbes après le parricide et l'inceste d'CEdipe, c'est la « terre gaste » du Moyen Âge, le terrible châtiment collectif, la stérilité de toute une région. Ces criminels sexuels rejoignent donc tous les personnages " monstrueux » des légendes: car "l'humanité normale, celle qui

Cf. Mét., 581-582.

Mét., 585-629, O. m., 2387-2473.

Cf. Mét.: meos furores (602), nefandum (626: c'est proprement ce qui ne doit pas arriver à la parole, le sacrilège, le tabou), crimina (629). O. m.: le mal qui m'afole (2390), ma fole pensee (2411), forsenage (2421), maladie, destresce, doulour (2428-30), desverie (2457), coupable (2464).

"Épuisée de larmes, elle se change en une fontaine ", Mét., IX, 663, loc. cit., p. 115.

Voir le chapitre sur "Le plaisir de conter" dans notre ouvrage LOvide moralisé. Essai d'interprétation, Paris, 2006, p. 235-256.

Cf. les vers 1 à 14 du Livre I avec notamment l'évocation de la Parabole des Talents, véritable topos dans les prologues médiévaux.

Dans Les deux seurs et leur mère, Paris, 1994. 
fait le semblable, c'est d'abord celle où l'espèce se reconnait, celle qui s'accouple comme nous, mange comme nous, a un langage organisé, et où tous les individus sont pourvus d'une identité constante, définitive ${ }^{28}$ ». Le scandale naît de l'existence, de la rencontre de celui qu'Aristote appelle «le monstre ». C'est pourquoi, si les histoires d'inceste sont horribles, elles rejoignent « la thématique du mutisme brutal ressenti par l'être humain qui se sent transformé en bête. Et l'effroi d'Actéon impuissant à avertir ses chiens qu'ils sont en train de dévorer leur maitre, celui des victimes de Circé, les compagnons d'Ulysse, horrifiés par les grognements qu'ils s'entendent émettre, sont dépeints avec une précision aussi suggestive que la souffrance des victimes féminines ${ }^{29}$ ".

Les " mauvaisetés " sexuelles - l'inceste tout particulièrement - sont condamnées dans toutes les civilisations. L'Ancien Testament lui-même les stigmatise: le chapitre 18 du Lévitique condamne l'inceste dans les versets 6 à 18. Les châtiments de ces fautes sont énoncés au chapitre 20 (versets 10 à 21). L'inceste est puni de mort. Le discours de conclusion du Deutéronome, au chapitre 27 (versets 20-22), annonce les fléaux qui doivent punir les délits sexuels. F. Héritier note les mêmes condamnations dans le Talmud ou le $\operatorname{Coran}^{30}$. Cette condamnation unanime a des racines très profondes, liées à "l'inconscient collectif " que révèle le mythe.

Françoise Héritier commente la célèbre légende de Philoména. L'amour de Térée pour Philoména comporte cet aspect incestueux: elle est sa belle-sœur. F. Héritier a défini cet inceste comme celui " du deuxième type ". L'inceste " du premier type " réunit sexuellement deux consanguins de sexe opposé ${ }^{31}$, comme Myrrha et son père, ou Byblis et son frère. L'inceste " du deuxième type » unit « des consanguins de même sexe qui ne sont pas homosexuels mais partagent le même partenaire sexuel ${ }^{32}$ ".

C'est ce délit que l'auteur de l'Ovide moralisé dénonce quand il appelle manvestié le désir de Térée pour Philoména: Pechiez le met an esperance / De mauvestié et de folie; I Amors vilainement le lie. I - Vilainement? - Voire, sans faille: / De vilenie se travaille, / Quant il son cuer viaut atorner / A la seror sa fame amer (VI, 2426-34). Ovide qualifie ce désir d'" effréné ", effreno (VI, 465), de " crime ", crimen (474), de " fureur ", furor (480).

F. Héritier justifie cette interdiction de l'inceste du deuxième type par «l'horreur du même »: Philoména et Procné, en tant que sœurs, partagent la même substance (celle de leur mère); en s'unissant au même homme, elles font se rencontrer cette même substance. Il n'y a pas d'autre raison, pour F. Héritier, à la honte de Philoména: à cause

Ibid.

Sourate IV Sur les femmes, versets 26 et 27.

Dans son "premier degré ", une mère et son fils, ou un père et sa fille; dans son " second degré ", un frère et sa sœur.

Cf. Lévitique 20, 14: "L'homme qui prend pour épouses une femme et sa mère: c'est un inceste. On les brûlera, lui et elles "Cf. versets 11 (femme du père) et 12 (femme du fils). 
de Térée, « elle a commis un inceste avec sa sœur », elle est devenue sa "rivale », et " lui l'époux de deux sœurs ${ }^{33}$ ». Dans le Lévitique, le châtiment est la mort.

L'auteur de l'Ovide moralisé précise cependant que cette union n'était pas proscrite dans la civilisation thrace - il est vrai que chez certains peuples, un homme avait même le devoir d'épouser la veuve de son frère, mais après sa mort. De cette façon, d'après F. Héritier, il ne pouvait y avoir dans l'épouse mélange de la substance des deux frères ${ }^{34}$.

L'interdiction de l'inceste relève aussi de ce que les ethnologues appellent l'exogamie: si le père ne garde pas pour lui sa fille, qui pourtant lui reviendrait de droit, c'est qu'il veut obtenir une femme pour son fils. La femme a donc une valeur d'échange.

Selon Georges Bataille, "le père doit faire entrer la richesse qu'est sa fille, le frère, celle qu'est sa sœur, dans un circuit d'échanges cérémoniels: il doit la donner en cadeau, mais le circuit suppose un ensemble de règles admises en un milieu donné comme le sont des règles de jeu 35 ». "Ainsi les femmes apparaissent-elles essentiellement vouées à la communication, entendue dans le sens fort du mot, le sens de l'effusion [...]. Le frère donnant sa sœur nie moins la valeur de l'union sexuelle avec celle qui lui est proche, qu'il n'affirme la valeur plus grande de mariages unissant cette sœur avec un autre homme, ou lui-même avec une autre femme. Il y a communication plus grande, dans l'échange à base de générosité, que dans la jouissance immédiate ${ }^{36}$. " G. Bataille rappelle la conclusion de Cl. Lévi-Strauss: "La prohibition de l'usage sexuel de la fille ou de la sœur contraint à donner en mariage la fille ou la sœur à un autre homme et, en même temps, elle crée un droit sur la fille ou la sœur de cet autre homme ${ }^{37}$. "

Or nous avons ce soupçon de relation incestueuse entre père et fille dans deux légendes au moins: si Daphné, si Philoména méprisent l'amour des hommes, nous devons comprendre qu'elles rejettent l'exogamie, et qu'elles lui préferent l'endogamie, comme semblent le prouver les caresses, l'intimité de leurs relations avec leur père.

Chez Ovide déjà, Daphné "suspend ses bras caressants au cou de son père ", Inque patris blandis haerens cervice lacertis (I, 485). Dans la translation romane, Son pere estroitement embrase, / Si dist: "Biau pere, je n'ai soing / De baron prendre ne besoing... " (I, 2844-46). Dans les deux versions, le père est responsable de la métamorphose en laurier de Daphné, qui garde ainsi sa virginité.

Philoména quant à elle est si proche de son père qu'il refuse d'abord de la confier à Térée; dans le texte latin, Philoména joint ses prières à celles de Térée et cajole son père, provoquant désir et jalousie chez Térée: «Quand il voit les baisers qu'elle

F. HÉRITIER, Les deux søurs, p. 53.

34 Le Talmud insiste sur le cas de l'union d'un homme avec deux sœurs: elle est impossible après le divorce ou la répudiation, mais possible seulement après la mort de la première femme (F. Héritier, Les deux sours, p. 84).

37 Structures élémentaires de la parenté, Paris, 1949, p. 65, cité par G. Bataille, L'érotisme, p. 233. 
donne et ses bras jetés autour du cou de son père, [...] il voudrait être son père, car il n'en serait pas moins mauvais parent ${ }^{38}$ " - "il n'en serait pas moins impie ", dit le latin, qui fait donc ici une allusion claire à l'inceste père-fille. Mais le Pandion latin alors "se laisse vaincre par les prières de ses deux filles ". Dans le texte roman, il faut trois discours de Térée à Pandion, trois prières successives pour que le roi d'Athènes se résigne à laisser partir sa fille. Après la première, Philoména (qui refuse de cajoler son père), est pessimiste: Bien sai que mes sire li rois / N'a talant que congié me doingne. / Ne li plest pas ceste besoingne (2520-22). Après la deuxième demande de Térée, c'est Pandion lui-même qui marque sa désapprobation: Pandions sur sa main s'apuie, / Cui ceste chose mout enuie. / Enuie qu'enoiier li puet, / Mes a respondre li estuet (2251-54): il est ennuyé d'être ennuyé, ce qui prouve bien que ses sentiments ne sont pas tout à fait avouables... Après avoir rappelé à Térée qu'il lui donnerait tout ce qu'il a s'il en avait besoin - il lui a déjà donné Procné, sa fille aînée, il ajoute: Mes je croi, se vos saviiez / Les biens que ma fille me fet, I Ja ne me metriiez an plet / De ce don vos me requerez. I Trop seroie desesperez / S'un jor estoie sanz ma fille (2562-65) : est-ce avoir l'esprit mal tourné que supposer que le translateur médiéval garde ici la trace d'une possible relation incestueuse entre Philoména et son père? Sans doute, les vers 2580-94 décrivent sans arrière-pensée un amour et des soins relevant de la plus pure piété filiale: Philoména aide son père à se vêtir, à se chausser, elle le soutient, le sert, l'aide à se coucher et à se lever. Mais l'allusion à l'inceste était explicite chez Ovide; elle est peut-être implicite dans la translation romane.

À propos de l'érotisme en général et des «mauvaisetés » sexuelles en particulier, G. Bataille évoque aussi ce rejet de l'animalité qui caractérise à ses yeux les relations humaines: "L'homme se dégagea de l'animalité première » « en travaillant, en comprenant qu'il mourait et en glissant de la sexualité sans honte à la sexualité honteuse, dont l'érotisme découla ${ }^{39}$. "C'est alors qu'il frappa d'interdits les actes sexuels. L'érotisme naît de la transgression de ces interdits. Mais «la transgression differe du "retour à la nature": elle lève l'interdit sans le supprimer ${ }^{40}$ ".

Cette façon de se distinguer de l'animalité, consécutive à l'idée que l'homme, «fait à l'image de Dieu ", est supérieur aux autres créatures, explique ainsi pour G. Bataille l'interdiction de l'inceste: «La renonciation (du père à sa fille, du frère à sa sœur), [...] que l'interdit fonda, a seule rendu le don possible. [...] et l'essence de l'humanité se dégage de ce dépassement. Le renoncement du proche parent - la réserve de celui qui s'interdit la chose même qui lui appartient - définit l'attitude humaine, tout à l'opposé de la voracité animale. [...] il contribue à créer le monde humain, où le respect, la difficulté et la réserve l'emportent sur la violence. [...] L'interdit ne change pas la violence Videndo / Osculaque et collo circumdata bracchia cernens, [...] / Esse parens vellet; neque enim minus impius esset, trad. et éd. cit., VI, 478-482, t. II, p. 18. G. Bataille, L'érotisme, p. 37. Ibid., p. 42. 
de l'activité sexuelle, mais il ouvre à l'homme discipliné une porte à laquelle l'animalité ne saurait accéder, celle de la transgression de la règle ${ }^{41}$."

Ces " mauvaisetés " sont donc condamnées parce qu'elles ravalent l'homme au rang des bêtes: Myrrha, dans son long monologue, envie le comportement sexuel des animaux, coeunt animalia nullo / Cetera delicto..., " tous les autres animaux s'accouplent sans choix ", Humana malignas / Cura dedit leges et quod natura remittit / Invida iura negant, "Les scrupules de l'homme ont créé des lois méchantes et ce que la nature permet, des arrêts jaloux le défendent ${ }^{42}$. " La loi et li drois nous desvoie / Ce que nature nous otroie: il est clair que ces relations portent atteinte aux règles de la cité, à l'ordre établi. Byblis, de la même façon, rejette ces lois dont le respect est symbolisé par les figures des vieillards: Iura senes norint, et, quid liceatque nefasque / Fasque sit, inquirant legumque examina servent, "Laissons aux vieillards la science du droit; à eux de rechercher ce qui est permis, ce qui est crime et ce qui ne l'est pas; à eux d'observer les balances de la loi " (IX, 551-552). L'Ovide moralisé traduit fidèlement, aux vers 22982302: Li viellart sachent que est droit. / Cil doivent la loi maintenir / Et eulz selonc droit contenir. / Contre droit ne doivent riens faire. / Ce n'apartient à nostre afere. On conçoit les risques d'une telle confusion.

Cependant, ce qui fait l'originalité de l'Ovide moralisé, c'est le renversement total qui peut s'opérer du récit de la légende à son interprétation: or, dans le cas de ces légendes d'amours incestueuses, on peut parler d'une véritable révolution.

\section{La réhabilitation chrétienne: incarnation et conversion}

Comme l'écrit David Hult, "l'auteur de l'Ovide moralisé accentue le rapport entre le christianisme et les fables ovidiennes les plus scabreuses lorsqu'il déclare que la naissance du Christ est elle-même "contre nature", expression dont il se sert également pour parler de l'avortement, de la bestialité [...], de l'homosexualité, [...], ou de l'inceste $[\ldots]^{43}$ ".

La grande "révolution » qu'opère le penseur chrétien, c'est de réhabiliter ces récits en leur donnant une signification positive, c'est de sublimer ces amours en les interprétant comme des figures de la charité chrétienne ${ }^{44}$. La justification véritable de cette complaisance du moraliste à retracer les légendes de " mauvaiseté » sexuelle, est en effet constituée par les interprétations par lesquelles il rend ces récits acceptables. Les fables obscènes sont réhabilitées par les allégorèses.

$41 \quad$ Ibid., p. 242-243.

42 Ovide, Mét., X, 324-331. O. m. X, 1181-89.

43 Dans "Allégories de la sexualité dans l'Ovide moralise", Lectures et usages d'Ovide (XIII $X V^{\ell}$ siècles): quelques approches, Cahiers de recherches médiévales 9 (2002), p. 65.

44 Ce qui, selon nous, témoigne de son appartenance à la mouvance franciscaine. Voir notre ouvrage LOvide moralisé. Essai d'interprétation, p. 749-788. 
La merveille chrétienne, c'est l'Incarnation, alliance contre nature entre Dieu et l'homme, preuve de l'amour inimaginable de Dieu pour sa créature. L'interprétation fait ainsi des récits d'amours incestueuses une représentation adéquate de l'amour réciproque de Dieu et de la créature humaine.

Myrrha conçoit Adonis de son union avec son père. L'Ovide moralisé livre d'abord une interprétation physique (reprise à Fulgence) qui glisse vers l'explication morale ${ }^{45}$. Mais il y a ensuite une autre sentence [...] / Mieudre et plus digne de savoir (X, 3748-49), interprétation typologique selon laquelle l'héroïne est la Vierge Marie, et Adonis le Christ. L'amour contre nature de Myrrha pour son père la prédispose en effet à devenir la figure de la Vierge, qui, comme l'héroïne, fu ardaument esprise / De l'amour Dieu, cui fille elle iere (3757-58). Cet amour est " contre nature " car il dépasse le cadre des amours terrestres:

La soie amour fu ferme et fine

Si parfete et si enterine

C'onques si fine amour ne fu.

Pour l'amour Dieu mist en refu

Toutes terriennes amours (X, 3762-66).

La nourrice qui favorisa les amours de Cynaras et de sa fille est la figure de l'humilité de la Vierge ${ }^{46}$, qui permit la merveille de l'Incarnation, la poison fort / De salu plaine et de confort, / De quoi Dieu se vault enmurer, / Pour touz reëmbre et delivrer (3786-89). La métaphore du sein de la Vierge comme "mur » qui enferma le Christ est appelée bien sûr par la métamorphose de Myrrha en arbre avant la naissance de son fils.

Au livre IX, la fable de Byblis reçoit elle aussi deux interprétations: Istorial sens puet avoir / La fable et bien puet estre voir (2531-32): Byblis aima son frère, qui dut s'enfuir; elle se livra à la prostitution, ce que la fable figure par sa transformation en fontaine ${ }^{47}$. Mais Sentence y a mieudre et plus saine, nous dit le vers 2550 pour introduire la lecture spirituelle, typologique aussi: Cadmus note l'umain lingnage, cette interprétation explique l'apparente confusion de notre auteur entre Caunus et Cadmus (l'équivalence entre Cadmus et l'humain lignage est constante depuis le livre III, et la " correction » de la fable préparait cette correspondance). Byblis quant à elle figure la divine sapience (2586):

45 Cynaras est le soleil, que la myrrhe aime plus que les autres arbres. De la gomme qui sort de la myrrhe, Qui douce oudour et souef rent, on fabrique un piment Qui trop est de chaude nature / Et done appetit de luxure: si la fable raconte que Vénus fut éprise d'Adonis, c'est que la luxure aime la beauté (X, 3678-3747).

46 Cette qualité est soulignée par les Franciscains, autre argument pour soutenir que l'auteur anonyme était un frère Mineur.

47 IX, 2544-49: Ains s'abandona par putage / A touz homes comunement. / Chascuns habandoneement / Pooit en lui puisier et prendre, / Sans contredire et sans deffendre, / Ainsi com l'en puise en fontaine. 
C'est la sapience devine,

Qui tout fist et par sa pitié

Mist tant en home s'amistié,

Tant li plot, tant li abelit,

Qu'il tint à souverain delit

De soi joindre à l'umain lignage

Par fraresche et par mariage.

D'umain lignage fist son frere

Cele qui crierresse et mere

Estoit de toute creature (IX, 2598-2607).

L'amour incestueux de l'héroïne pour son frère est une représentation concrète de l'amour incroyable du Créateur pour sa créature: Cele se vault contre nature / Charnelment à homme assambler / Et creature resambler (2608-2610). Sa métamorphose en fontaine figure la fontaine nete et pure / Qui au monde a vie rendue (2660-61): la dévotion de l'auteur du XIve siècle pour la Passion du Christ s'exprime ici par une anaphore du mot fontaine.

C'est la fontaine droitement

Dont sourt la pardurable vie

Et qui les bevans vivifie.

Diex est fontaine nete et pure,

Espurgement de toute ordure,

Fontaine douce et delitable,

Fontaine vive et pardurable,

Que nulz ne porroit espuisier,

Combien qu'il y vausist puisier (IX, 2664-74).

Mais une autre " métamorphose " chrétienne exige la même radicalité: celle qui ramène la créature à son Créateur, celle par laquelle l'âme humaine rend à Dieu l'amour dont il la comble: la conversion. Cette métamorphose totale, ce retournement, peut se faire par le moyen des métamorphoses du cœur humain que suppose l'observance des sacrements, en particulier le sacrement de pénitence.

Dans l'interprétation de la légende de Myrrha, une deuxième leçon semble contredire la première lecture. Elle est pourtant assez samblable à voir (X, 3811). C'est une interprétation tropologique après la sentence typologique. Myrrha devient l'image de l'ame pecheresse et honie (3813) qui communie en état de péché (l'autel est compris comme la couche / Ou li cors Dieu repose et couche, 3822-23). Mais la figure de Myrrha est sauvée par son repentir final: après un passage par la figure typologique de MarieMadeleine (3897-3916), l'interprétation s'appuie sur la métamorphose en myrrhe de l'héroïne mythologique, et sur les propriétés naturelles de la plante, qui empêche la putréfaction des chairs mortes. Le repentir et la réparation, deux des aspects du 
sacrement de pénitence, sont illustrés par le personnage de la fable et se répondent à la rime:

Mes elle s'oint de tele ointure

Qui mort garde de porreture,

C'est d'amere compocion

O douce satifacion,

Qu'ele ot tant come elle vesqui (X, 3932-36).

En transcrivant sans les édulcorer les fables scandaleuses, le penseur chrétien se fait l'écho de la pensée mythique et des réflexions moralistes de toutes les sociétés patriarcales, c'est-à-dire occidentales, de l'Antiquité et du Moyen Âge. Il condamne certes, au moment de la translation de ces légendes, les comportements sexuels tels que l'inceste, du premier et du deuxième type, parce qu'ils menacent l'ordre établi ou ravalent l'homme au rang des bêtes.

Mais l'Ovide moralisé dépasse ce premier niveau d'interprétation. La merveille du texte, c'est la métamorphose chrétienne, l'interprétation si troublante qu'elle peut provoquer chez les lecteurs l'incompréhension et l'accusation d'incohérence: Myrrha l'incestueuse devient une figure de la Vierge, Byblis amoureuse de son frère est comprise comme le Christ. Les histoires scandaleuses de la mythologie antique figurent le mystère de l'Incarnation divine, l'alliance incroyable de la nature humaine et de la nature divine, ou le prodige de la conversion, le retournement de l'âme pécheresse vers la figure de Dieu. Par le biais de l'allégorèse chrétienne, ces récits sordides deviennent une image concrète du grand "scandale " de la foi chrétienne, l'amour inconcevable entre Dieu et sa créature. Ces interprétations, loin d'être absurdes, sont le vrai miracle de ce texte extraordinaire.

Marylène PossamaI-PÉREZ

Université Lyon 2-Lumière

Faculté LESLA

18, quai Claude Bernard

69365 Lyon cedex 07

marylenepossamai@aol.com 